\title{
Modelling liquid-liquid equilibria for island type ternary systems
}

\author{
M.M. Olaya, J.A. Reyes-Labarta, R. Velasco, I. Ibarra, A. Marcilla* \\ Dpto. Ingeniería Química, Universidad de Alicante, Apdo. 99, Alicante 03080, Spain
}

Received 22 November 2007; received in revised form 21 December 2007; accepted 28 December 2007

\begin{abstract}
Closed miscibility gaps in ternary liquid mixtures, at constant temperature and pressure, are obtained if phase separations occur only in the ternary region, whilst all binary mixtures involved in the system are completely miscible. This type of behaviour, although not very frequent, has been observed for a certain number of systems. Nevertheless, we have found no information about the applicability of the common activity coefficient models, as NRTL and UNIQUAC, for these types of ternary systems. Moreover, any of the island type systems published in the most common liquid-liquid equilibrium data collections, are correlated with any model. In this paper, the applicability of the NRTL equation to model the LLE of island type systems is assessed using topological concepts related to the Gibbs stability test. A first attempt to correlate experimental LLE data for two island type ternary systems is also presented.
\end{abstract}

(C) 2008 Elsevier B.V. All rights reserved.

Keywords: Island miscibility gaps; LLE; NRTL; Gibbs energy of mixing; Gibbs stability test

\section{Introduction}

In LLE island type ternary systems (type 0 in the Treybal classification [1]) at constant temperature and pressure, a binodal curve lies around a heterogeneous two liquid area (LL) that is entirely surrounded by a homogeneous one liquid area (L). Therefore, there is complete miscibility in each of the three binary systems involved, which simultaneously coexist with a LL ternary region. An island binodal curve has two plait points that limit the tie-lines on each side of the island [2].

Island binodal curves result commonly from the simultaneous presence of a weak acid and base, that reversibly form a soluble salt, which has a lower miscibility than either the free acid or the free base, leading to the liquid-liquid phase separation. For example, Becker and Richter [3] determined the binodal curve for island systems of the type: carboxylic acid + tertiary amine + hydrocarbon. Closed miscibility gaps may also occur in ternary systems of two polymers and a low molecular weight solvent [4].

On the other hand, it is a common practice to utilize a model to fit the experimental LLE data and obtain the binary interaction parameter values that can be used to calculate any tie-line,

\footnotetext{
* Corresponding author. Tel.: +34 965 903789; fax: +34 965903826.

E-mail address: antonio.marcilla@ua.es (A. Marcilla).
}

for example, as is required by commercial software packages for chemical process design. However, despite the experimental evidence of island type ternary systems is not so infrequent, very few papers have been found in the literature about the applicability or application of the common LLE models to these systems [5,6]. Wilson [7] and NRTL [8] equations with a ternary correction, were applied by Řehák et al. [5] for the correlation of the phenol + tetrahydrofuran + water system. Novák et al. [6] used the regular-solution model with temperature-dependent parameters to describe critical curves of LLE in ternary systems where the possibility for the appearance of a closed heterogeneous region with two critical points was discussed. It is also quite relevant that none of the island type systems compiled in liquid-liquid equilibrium data collection, DECHEMA Chemistry Data Series [9], is correlated, whilst, however, the NRTL and UNIQUAC [10] binary parameters are given for all the type I and II ternary systems (Treybal classification [1]). These facts might suggest that local composition models as NRTL or UNIQUAC are not able to predict a ternary LL region comprising the three completely miscible binary systems. However, no discussion of this matter has been found in the literature. The DECHEMA Data Preparation Package (DPP, DECHEMA e.V.), a very useful software package for the regression of thermophysical properties, has also been evaluated on its ability to regress LLE data for island type ternary systems. The outcome is that, even though this tool is very flexible for the LLE regression of 
type I and II ternary systems, the results obtained are poor when it is used for type III systems, and it is not able to carry out calculations for island type systems, because the program requires the existence of at least one partially miscible pair of compounds.

The authors of the present paper have analyzed the limitations of local composition equations as NRTL to model the LLE behaviour of many systems. For example, most of the type III ternary systems cannot be correlated using NRTL or UNIQUAC models [11]. Our study was also concerned with the simultaneous correlation of all the equilibrium regions for ternary systems containing an inorganic salt, which is of interest due to the salting-out effect [12]. In dealing with these, and other similar problems related to liquid-liquid (LL), liquid-solid (LS) and liquid-liquid-solid (LLS) equilibrium calculations, we always consider the importance of the topological analysis of the Gibbs energy of mixing function $\left(\Delta_{\text {mix }} G / R T\right.$ and, hereafter, $\left.g^{\mathrm{M}}\right)$ related to the equilibrium condition based on the Gibbs tangent plane criterion (Gibbs stability test). Using this approach, we have studied what the characteristics required for the Gibbs energy of mixing surface must be to simultaneously fit all the equilibrium regions for water + organic solvent + inorganic salt ternary systems, where LL, LS and LLS equilibrium zones exist [13].

Obviously, island type ternary systems require that the three binary $g^{\mathrm{M}}$ curves correspond to homogeneous (L) behaviour (Fig. 1(a)), for which there is no possibility of finding two points on the $g^{\mathrm{M}}$ curve with a common tangent line. A ternary region must also exist where the Gibbs energy of mixing surface permits the existence of conjugated points with a common tangent plane, which must not intersect the surface at any other composition either. For example, in Fig. 1(b) a cross-section of the aforementioned Gibbs energy surface is shown where two conjugated points with a common tangent line lead to the formation of two liquid phases (LL). Any initial mixture as $z_{i}$ in Fig. 1(b), located between the two inflection points on the $g^{\mathrm{M}}$ curve, is thermodynamically unstable and splits into two liquid phases having the compositions $x_{i}^{\mathrm{I}}$ and $x_{i}^{\mathrm{II}}$, and a lower value of the overall Gibbs energy of mixing.

In this context, the question of why the NRTL model was not used to correlate the LLE data for island type systems arose, so that a systematic analysis of the flexibility of the NRTL model to generate binodal curves for such systems was planned. To this end, the topological concepts related to the Gibbs energy of mixing and the Gibbs tangent plane criterion formulated by Gibbs (stability test), and generalized by Smith et al. [14] and Jiang et al. [15], have been used. After the potential capability of and the requirements for the NRTL equation to model island type ternary systems were evaluated, the correlation of some experimental LLE data for such systems was carried out.

\section{Systematic analysis of the NRTL $g^{\mathrm{M}}$ surface}

\subsection{Procedure}

The three binary $g^{\mathrm{M}}$ curves obtained, using the NRTL equation for the 1-2-3 ternary system, have been systematically varied and, for each new situation, the ternary LLE data were calculated, looking for the appearance of an island binodal curve. The temperature was set at $25^{\circ} \mathrm{C}$. To guarantee that binary liquid-liquid splitting did not occur, as is required for island type ternary systems, the empirical relation among the NRTL binary parameters reported elsewhere [13] was used.

The homogeneous binary $g^{\mathrm{M}}$ curves were characterized using two parameters: the composition of the minimum $\left(x_{i, \min }\right)$ and its corresponding energy value $\left(g_{\text {min }}^{\mathrm{M}}\right)$ as represented in Fig. 1(a). These two parameters were arbitrarily set and systematically varied. For each pair of values $\left(x_{i, \min }, g_{\text {min }}^{\mathrm{M}}\right)$, the NRTL $g^{\mathrm{M}}$ curve is defined at constant temperature and pressure. Therefore, we calculated the values of the two binary interaction parameters, $A_{i j}$ and $A_{j i}$, corresponding to that $g^{\mathrm{M}}$ curve using the following equations:

$g^{\mathrm{M}}\left(x_{i, \min }\right)=g_{\mathrm{min}}^{\mathrm{M}}$

$\frac{\mathrm{d} g^{\mathrm{M}}\left(x_{i, \min }\right)}{\mathrm{d} x_{i}}=0, \quad \frac{\mathrm{d}^{2} g^{\mathrm{M}}\left(x_{i, \min }\right)}{\mathrm{d} x_{i}^{2}}>0$

For the topological analysis, we have evaluated a wide range of $x_{i, \min }$ and $g_{\min }^{\mathrm{M}}$ values which return the values of $A_{i j}$ (NRTL) shown in the Appendix A in Tables A1-A4. The range of $g_{\text {min }}^{\mathrm{M}}$ values are from -0.2 to -5 , which correspond to normal values for the two $A_{i j}$ and $A_{j i}$ NRTL binary parameters (from -2500 to 2500), always considering only those values that return an homogeneous $g^{\mathrm{M}}$ curve, as shown in Fig. 1(a). The reason for the jumps in the $g_{\min }^{\mathrm{M}}$ values shown in the tables is the existence of gaps where it is not possible to find homogeneous $g^{\mathrm{M}}$ curves.
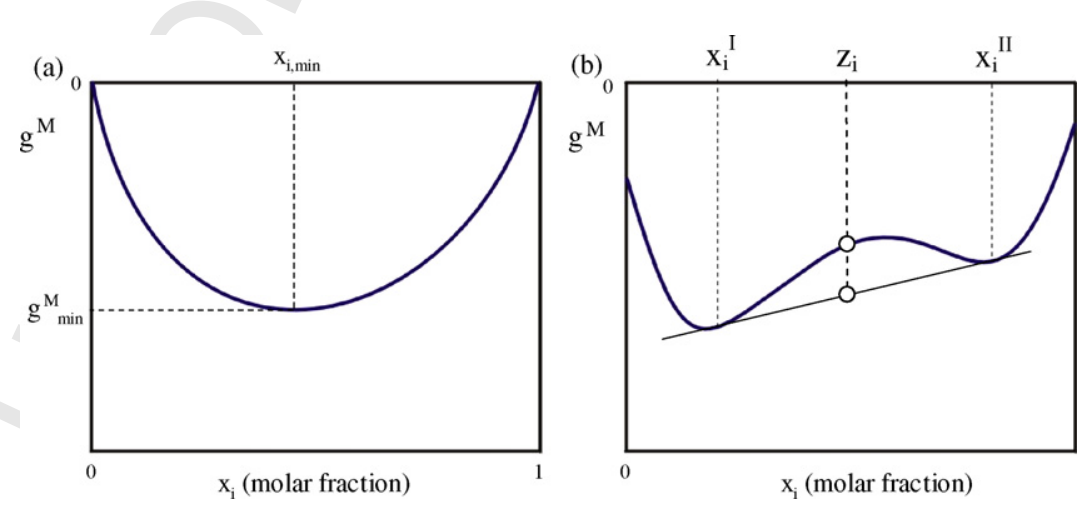

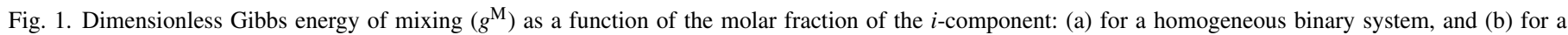
heterogeneous ternary system (LL) (cross-section). 
In this study, values for $x_{i, \min }$ vary from 0.35 to 0.65 because values closer to 0 and 1 could not be found without phase splitting occurring for any value of $g_{\min }^{\mathrm{M}}$. In Tables A1-A4 only values for $x_{i, \min }$ from 0.35 to 0.50 have been included because the symmetry of the NRTL model permits the construction of equivalent tables for $x_{i, \min }$ from 0.50 to 0.65 by changing the order of the indexes of the parameters.

Three $g^{\mathrm{M}}$ binary curves were systematically combined to obtain ternary systems. To do this, its corresponding set of six NRTL binary parameters were used to obtain the ternary LLE data through isothermal flash calculations, looking for the appearance of island type binodal curves.

\subsection{Results}

\subsubsection{Requirements for the $g^{M}$ binary curves to obtain an island type ternary system}

In accordance with the systematic study that has been carried out, the NRTL model is able to generate island type ternary systems, but only under a restrictive condition: the $g^{\mathrm{M}}$ curve for one of the three binary systems included in the ternary system must be much larger, or in other words, more negative (dissimilar binary $g^{\mathrm{M}}$ curve) than the other two binary $g^{\mathrm{M}}$ curves.

As an example, Fig. 2 shows a $g^{\mathrm{M}}$ surface calculated using the NRTL model that corresponds with the behaviour of the island type ternary system. A tangent line indicates the part of the surface where conjugated points with a tangent plane can be found, thus establishing formation of the ternary island LL region that has been represented on the triangular base of the prism shown in Fig. 2 (note that the binodal curve there represented does not touch the sides of the ternary composition diagram, although it is very close to them). In this example, the dissimilar binary is $1-2$, with a more negative minimum value $\left(g_{\mathrm{min}}^{\mathrm{M}}=-5.0\right)$ than the other two, 2-3 and 1-3 binaries, both with $g_{\min }^{\mathrm{M}}=-0.5$.

It has been observed that if this condition is disrupted, by either increasing the value of $g_{\min }^{\mathrm{M}}$ of the dissimilar binary, or by decreasing it for any of the other two binary systems, the island

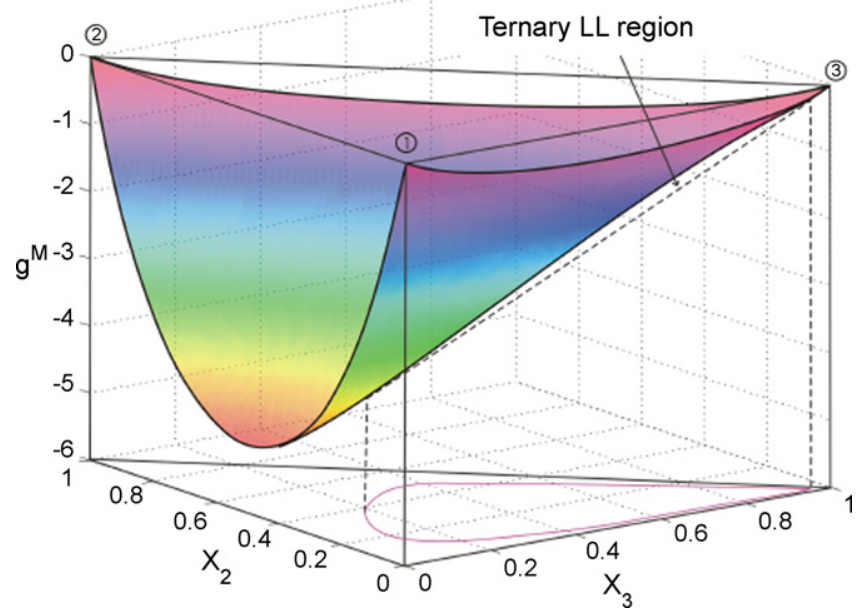

Fig. 2. An example of the $g^{\mathrm{M}}$ surface calculated using the NRTL model for an island type ternary system with the following binary parameters $(\mathrm{K})$ : $A_{12}=A_{21}=-1695.78$ and $A_{23}=A_{32}=A_{13}=A_{31}=120.00$.

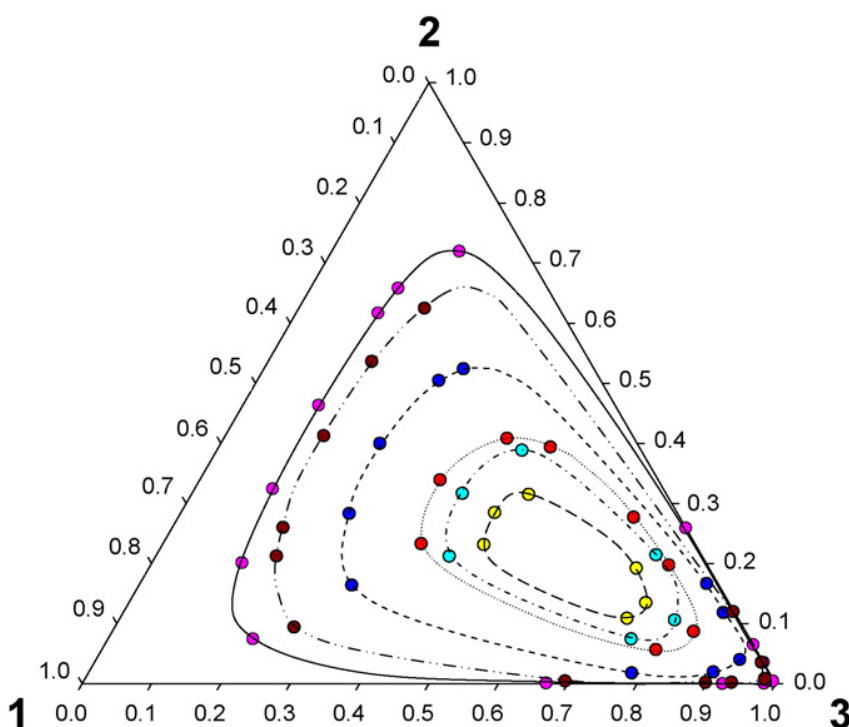

\begin{tabular}{|c|c|c|c|c|}
\hline & \multicolumn{2}{|c|}{ 1-2 BINARY } & \multicolumn{2}{|c|}{ NRTL PARAMETERS } \\
\hline & $\mathrm{g}_{\text {min }}^{\mathrm{M}^{\prime}}$ & $x_{2 \min }$ & $A_{12}$ & $A_{21}$ \\
\hline \multirow{6}{*}{$\begin{array}{c}-0 \\
-0 \\
0 \\
0 \\
-0 \\
-0 \\
-0 \\
0\end{array}$} & -2.3 & 0.50 & -765.72 & -765.72 \\
\hline & -2.4 & 0.50 & -805.38 & -805.38 \\
\hline & -2.5 & 0.50 & -844.45 & -844.45 \\
\hline & -3.0 & 0.50 & -1032.0 & -1032.0 \\
\hline & -4.0 & 0.50 & -1377.3 & -1377.3 \\
\hline & -5.0 & 0.50 & -1695.8 & -1695.8 \\
\hline
\end{tabular}

Fig. 3. Evolution of the island binodal curve when the $g_{\min }^{\mathrm{M}}$ for the 1-2 binary system is varied from -5.0 to -2.3 . For the other two binaries (1-3 and 2-3), $x_{i, \min }=0.50$ and $g_{\min }^{\mathrm{M}}=-0.5$.

curve disappears. As an example, Fig. 3 shows how the area inside the island binodal curve decreases when the $g_{\min }^{\mathrm{M}}$ for the $1-2$ binary system increases from -5.0 to -2.3 . For $g_{\min }^{\mathrm{M}}=-2.2$ and higher, the island type behaviour disappears and the ternary system is then completely homogeneous (L). This figure has been calculated with the minimum of the three $g^{\mathrm{M}}$ binary curves at $x_{i, \min }=0.5$ and $g_{\text {min }}^{\mathrm{M}}=-0.5$, but similar conclusions have been deduced for the minima located at different compositions.

\subsubsection{Influence of the position of the minimum of the binary $g^{M}$ curves $\left(x_{i, m i n}\right)$ on the location and size of the island binodal curve}

In cases where the values for the $g_{\min }^{\mathrm{M}}$ are fixed and the position of the minimum $\left(x_{i, \min }\right)$ is shifted for each one of the three binary systems, the model behaves as follows:

(a) When $x_{i, \min }$ of the dissimilar binary curve (1-2 in the present paper) is displaced in a given direction, the island curve is displaced in the same direction as $x_{i, \min }$ (Fig. 4).

(b) The opposite behaviour is observed when the $x_{i, \min }$ is displaced for the other two binary systems. When $x_{3, \min }$ is displaced in any of both binary systems $2-3$ or 1-3, the binodal island curve expands in the opposite direction. In this case not only a displacement, but mainly an increase in the island size occurs. Figs. 5 and 6 are used to illustrate this point for the displacement of $x_{3, \min }$ in binary systems $2-3$ and $1-3$, respectively. 


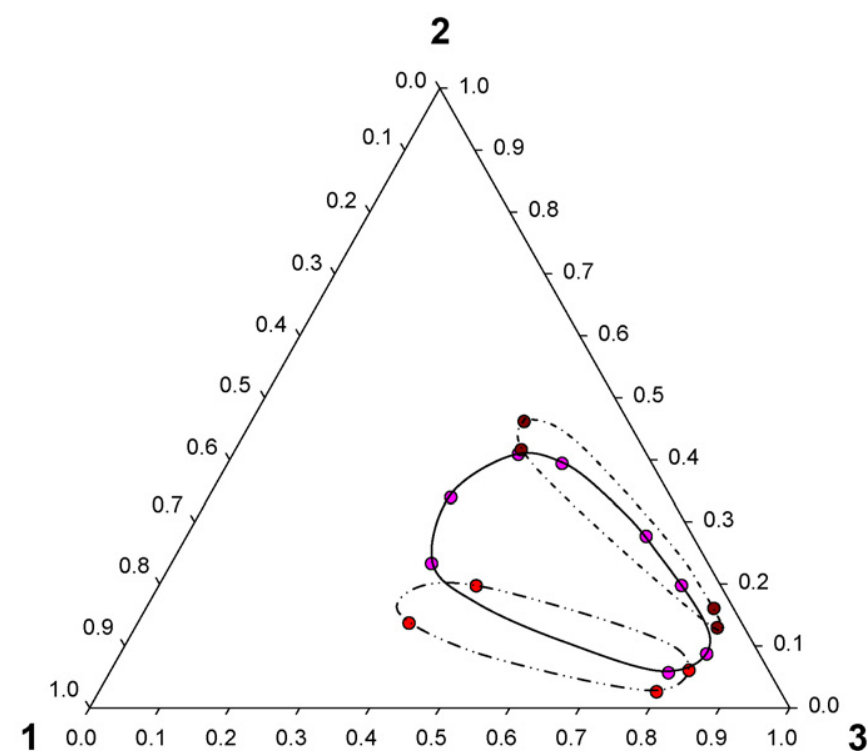

\begin{tabular}{|c|c|c|c|c|}
\hline & \multicolumn{2}{|c|}{ 1-2 BINARY } & \multicolumn{2}{|c|}{ NRTL PARAMETERS } \\
\hline & $g_{\text {min }}^{M_{1}}$ & $x_{1 \min }$ & $A_{12}$ & $A_{21}$ \\
\hline \multirow{3}{*}{$\begin{array}{l}-\theta- \\
-\theta \\
-\theta \cdots\end{array}$} & -2.5 & 0.40 & -1479.4 & 110.44 \\
\hline & -2.5 & 0.50 & -844.45 & -844.45 \\
\hline & -2.5 & 0.60 & 110.44 & -1479.4 \\
\hline
\end{tabular}

Fig. 4. Evolution of the island binodal curve when the $x_{1, \min }$ is displaced in the 1-2 binary system from 0.40 to 0.60 . For the other two binaries (1-3 and 2-3), $x_{i, \min }=0.50$ and $g_{\min }^{\mathrm{M}}=-0.5$.

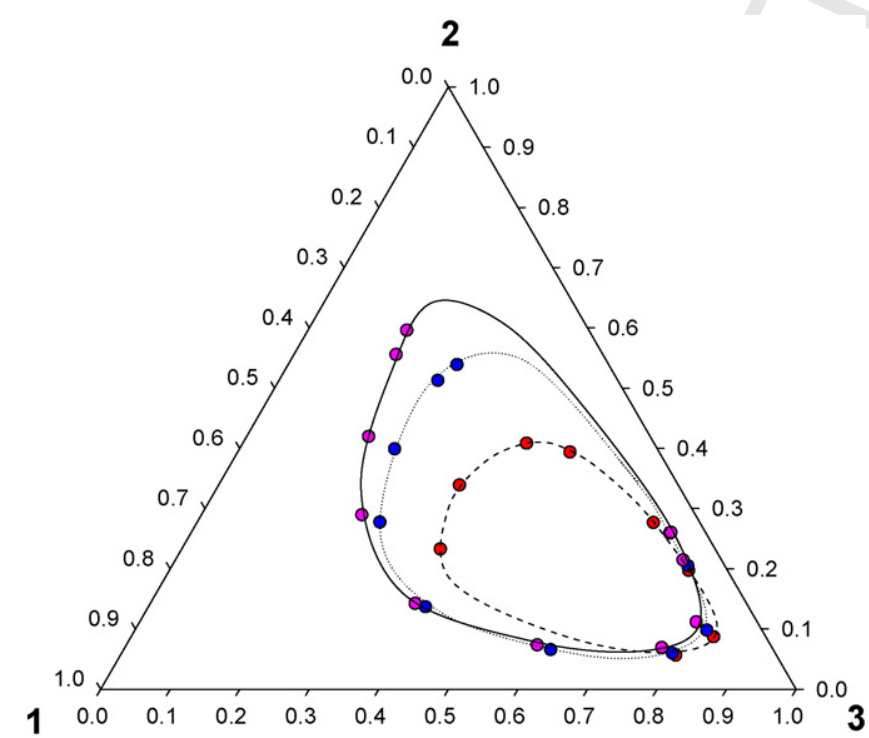

Fig. 5. Evolution of the island binodal curve when the $x_{3, \min }$ is displaced in the 2-3 binary system from 0.50 to 0.60 . For the $1-2$ binary, $x_{2, \min }=0.50$ and $g_{\min }^{\mathrm{M}}=-2.5$ and for the 1-3 binary, $x_{3, \min }=0.50$ and $g_{\min }^{\mathrm{M}}=-0.5$.

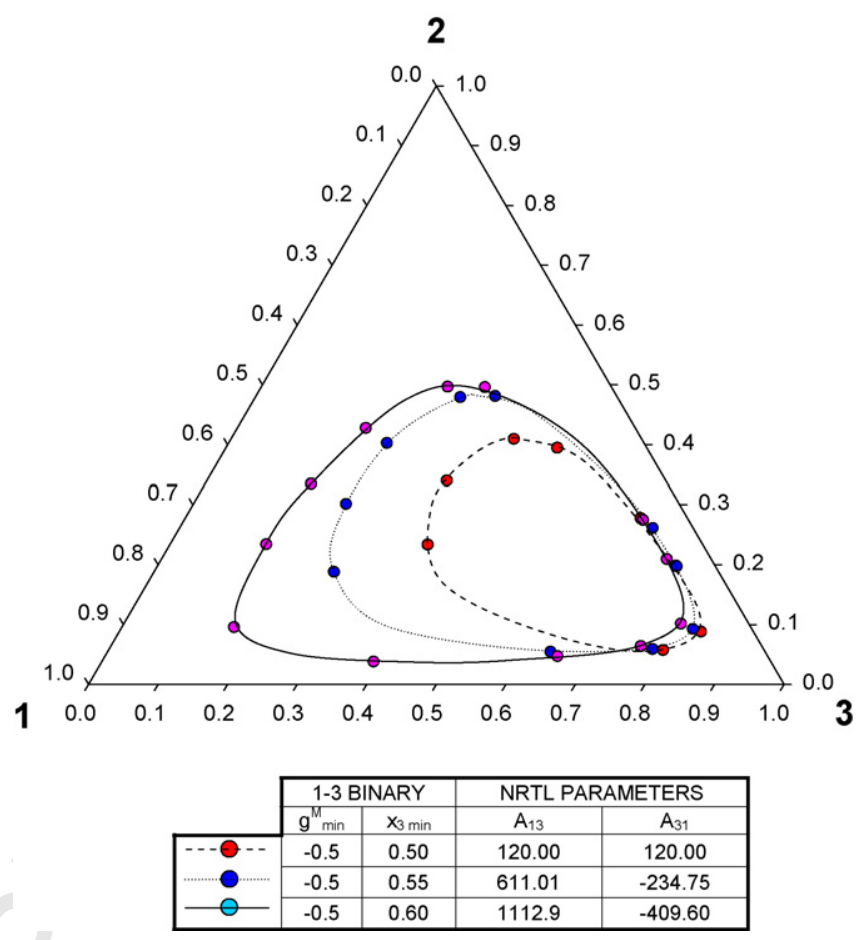

Fig. 6. Evolution of the island binodal curve when the $x_{3, \min }$ is displaced in the $1-3$ binary system from 0.50 to 0.60 . For the $1-2$ binary, $x_{2, \min }=0.50$ and $g_{\min }^{\mathrm{M}}=-2.5$, and for the 2-3 binary, $x_{3, \min }=0.50$ and $g_{\min }^{\mathrm{M}}=-0.5$.

\subsubsection{Restrictions on the location of the island binodal curve in the ternary diagram}

We have also checked that the island curves calculated using the NRTL model are preferentially located close to the opposite vertex of the dissimilar binary system. In other words, if 1-2 is the dissimilar binary system, the island curves calculated, having very different sizes and forms depending on the three $g^{\mathrm{M}}$ binary curves, are always preferentially located close to the 3component (Fig. 7). The requirement of a dissimilar $g^{\mathrm{M}}$ binary curve for an island type ternary system is not compatible with the existence of a centred and symmetric LLE ternary island.

\subsubsection{NRTL binary parameter values}

The requisites for ternary island type formation have been determined through the use of two properties, $x_{i, \min }$ and $g_{\min }^{\mathrm{M}}$, that define the three binary $g^{\mathrm{M}}$ curves of the ternary system. However, this information is of limited usefulness during the equilibrium data correlation because it is not directly applied in the fitting process, in which only the binary parameters of the model, and no other characteristic of the $g^{\mathrm{M}}$ binary curve, are optimized to find the best agreement between the experimental and calculated equilibrium data. For this reason, we thought it was very useful to directly convert the requirements previously deduced, to a function of the NRTL binary parameter values. As has been previously shown, the main requisites for LLE island formation is the existence of one dissimilar binary (1-2), together with complete miscibility of all three binary systems. All the island type systems systematically generated in this study satisfy the first condition for the 1-2 dissimilar binary curve with $g_{\text {min }}^{\mathrm{M}} \leq$ -2.3 , whereas for the $2-3$ and $1-3$ binary curves, $g_{\text {min }}^{\mathrm{M}}>-0.8$. 


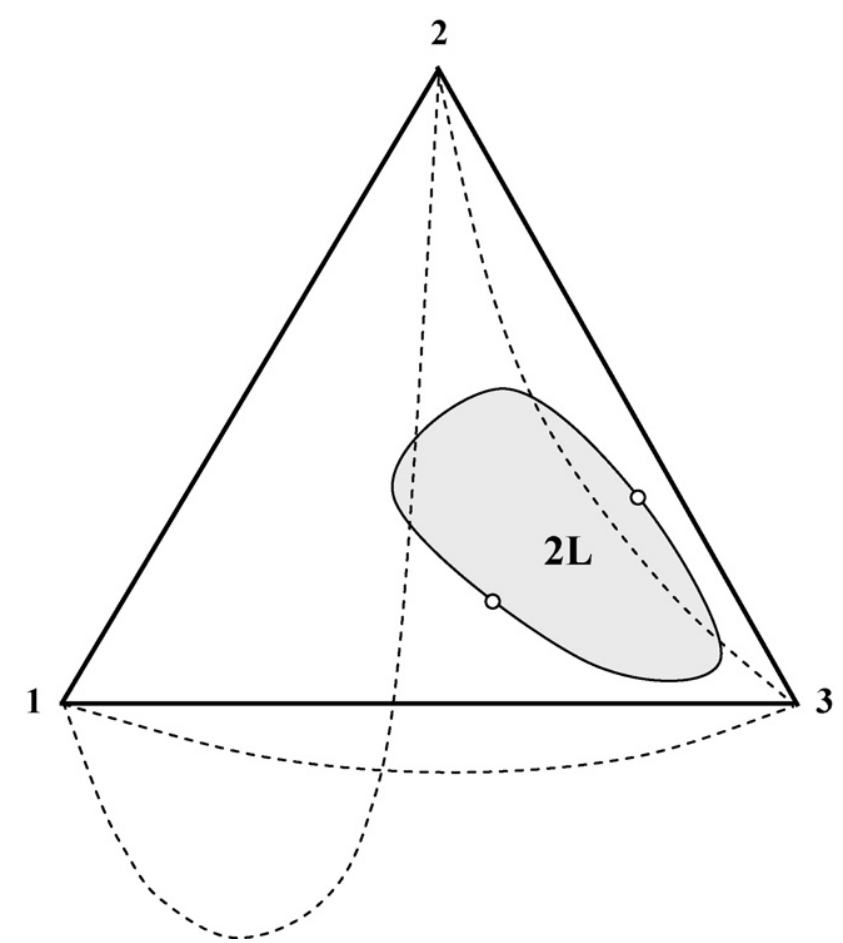

Fig. 7. Schematic representation to show that the island binodal curve is preferentially located close to the component opposite the dissimilar binary system (the 1-2 binary in this example): (- - -) binary $g^{\mathrm{M}}$ curves; (-) island type binodal curve; $(\bigcirc)$ plait point.

Fig. 8 shows the sum of both binary parameters $A_{i j}+A_{j i}$ versus $g_{\text {min }}^{\mathrm{M}}$ value for the $g^{\mathrm{M}}$ binary curve obtained using NRTL model for all the data in Tables A1-A4. From this figure, a requirement for the existence of an island type binodal curve may be deduced: the sum of the two binary parameters must be negative only for one of the three pairs included in the ternary system, and must be positive for the other two pairs:

$A_{12}+A_{21}<0$ (dissimilar binary pair)

$A_{13}+A_{31}>0$

$A_{23}+A_{32}>0$

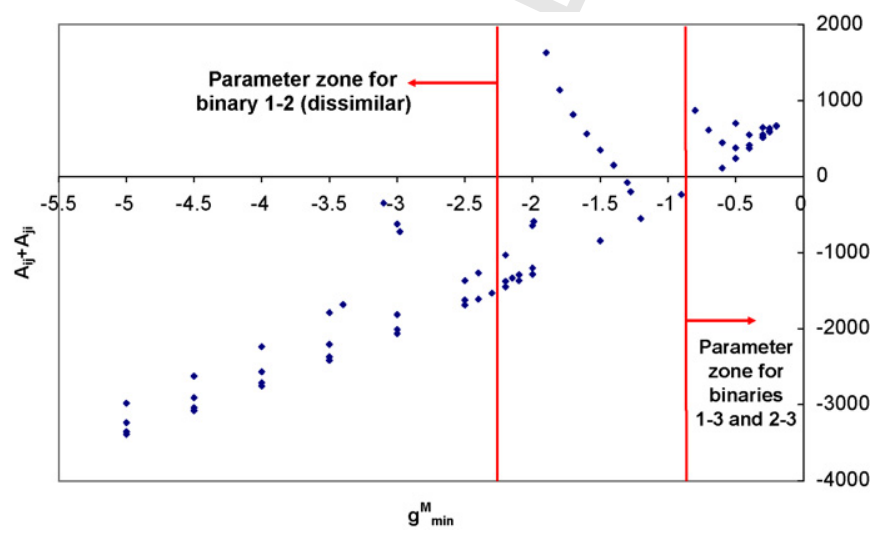

Fig. 8. The sum of the conjugated interaction binary parameters $A_{i j}+A_{j i}$ as a function of the $g_{\text {min }}^{\mathrm{M}}$ value, using the NRTL model $\left(\alpha=0.2\right.$ and $\left.T=25^{\circ} \mathrm{C}\right)$.
Obviously, this is an empirical condition derived from the behaviour observed in all of the almost 40 island type ternary systems generated in the systematic study carried out.

Usually, when LLE tie-lines for island type systems are regressed, the values of the parameters obtained as a result of the regression correspond with type I or type II ternary systems, where the one or two pairs of compounds are partially miscible, respectively. This result is not consistent with the real behaviour of the island system, which requires complete miscibility for all the binary pairs. These inconsistencies are encountered when commercial software, as for example DECHEMA Data Preparation Package and ChemCAD (Chemstations Inc.), are used for the regression of experimental LLE data for island type systems, because they are not prepare to deal with this type of systems. The conditions that we presented in a previous paper [13] about the values for the NRTL binary parameters which correspond to completely or partially miscible behaviour, can be used during the regression procedure to avoid inconsistencies. However, complete miscibility of all the binary pairs of the system is a necessary condition but not a guarantee of the existence of the LLE island gap. To guarantee existence of the ternary LLE island region, the practical restrictions represented by Eqs. (3)-(5) must be also fulfilled. All these conditions may be employed in the correlation method, as restrictions on the binary parameter values of the model, to guarantee that the final solution is consistent with island behaviour for the system, and also to guide the correlation process.

The study was carried out at $25^{\circ} \mathrm{C}$, but the practical condition derived from the values of the binary parameters to generate island type ternary systems (Eqs. (3)-(5)), are also valid for temperatures close to room temperature.

\section{Correlation of the LLE experimental data for island type ternary systems}

The correlation of the experimental LLE data for two island type ternary systems compiled in DECHEMA Chemistry Data Series has been carried out using the NRTL model. The systems are: water (1) + dimethyl sulfoxide (2) + tetrahydrofuran (3) at $20^{\circ} \mathrm{C}$, and trichloro acetic acid (1) + antipyrine (2) + water (3) at $30^{\circ} \mathrm{C}$.

The isopotential criterion has been used to calculate the LLE. The concentrations that satisfy the following equation are calculated:

$\sum_{k=1}^{n} \sum_{i=1}^{3}\left[\left(a_{i k}\right)_{\mathrm{or}}-\left(a_{i k}\right)_{\mathrm{aq}}\right]^{2}=0$

where $a_{i k}$ is the activity of component $i$ on the $k$ th tie-line; $n$ denote the number of tie-lines in the LL region; and or and aq represent the organic and aqueous phases, respectively. These concentrations are then substituted in Eq. (7) to obtain the composition objective function (O.F.) to be minimized:

O.F. $=\sum_{k=1}^{n} \sum_{i=1}^{3}\left[\left(x_{i k}^{\mathrm{exp}}-x_{i k}^{\mathrm{cal}}\right)_{\mathrm{or}}^{2}+\left(x_{i k}^{\exp }-x_{i k}^{\mathrm{cal}}\right)_{\mathrm{aq}}^{2}\right]$ 
Table 1

Correlation results using NRTL $(\alpha=0.2)$ for the water (1) + dimethyl sulfoxide (2) + tetrahydrofuran (3) system at $20^{\circ} \mathrm{C}$ : binary parameters $A_{i j}(\mathrm{~K})$, objective function (O.F.) and the standard deviation $(\sigma)$

\begin{tabular}{lcccc}
\hline & & \multicolumn{3}{l}{$A_{i j}(\mathrm{~K})$} \\
\cline { 3 - 5 } & & $j$ & 2 & 3 \\
\cline { 3 - 5 } & & 1 & 338.44 & 523.05 \\
& 1 & - & - & 1278.0 \\
& 2 & -1552.5 & -417.09 & - \\
\hline
\end{tabular}

O.F. $=0.143, \sigma=5.83$.

where $x_{i k}$ is the molar fraction of component $i$ on tie-line $k$; and exp and cal are the experimental and calculated equilibrium data, respectively. The optimization of the binary parameters $\left(A_{i j}\right)$ of the NRTL model has been done using the simplex flexible method.

To guarantee that the solution of the LLE data fitting corresponds to the island type ternary system, the restrictions on the parameters shown above were used (Eqs. (3)-(5)), together with the complete miscibility condition for all three binary systems [13]. In both systems, it was verified that if these conditions were not used, the final solutions of the optimization process were generally a type I or type II ternary system and, therefore, not consistent with the behaviour of the systems.

Tables 1 and 2 show the NRTL binary parameters $\left(A_{i j}\right)$ obtained from the fitting for both island type ternary systems. Standard deviations, calculated using the following equation, are also included:

$\sigma=100 \sqrt{\frac{\sum_{k=1}^{n} \sum_{i=1}^{3}\left(x_{i k}^{\mathrm{exp}}-x_{i k}^{\mathrm{cal}}\right)_{\mathrm{or}}^{2}+\left(x_{i k}^{\mathrm{exp}}-x_{i k}^{\mathrm{cal}}\right)_{\mathrm{aq}}^{2}}{6 n}}$

Experimental and calculated results are also graphically represented in Figs. 9 and 10. The parameters obtained in both regressions are clearly consistent with the island behaviour of these systems, as has been imposed by means of the parameter restrictions used in the optimization program. However, the regression results are poor, mainly for the trichloro acetic acid (1) + antipyrine (2) + water (3) system at $30^{\circ} \mathrm{C}$. The lack of flexibility of the NRTL model is responsible for the poor agreement between the experimental and calculated LLE data, highlighting the necessity for new models to be more flexible in and capable

Table 2

Correlation results using NRTL $(\alpha=0.2)$ for the trichloro acetic acid (1) + antipyrine (2) + water (3) system at $30^{\circ} \mathrm{C}$ : binary parameters $A_{i j}(\mathrm{~K})$, objective function (O.F.) and the standard deviation $(\sigma)$

\begin{tabular}{|c|c|c|c|c|}
\hline & & \multicolumn{3}{|l|}{$A_{i j}(\mathrm{~K})$} \\
\hline & & \multicolumn{3}{|l|}{$j$} \\
\hline & & 1 & 2 & 3 \\
\hline \multirow{3}{*}{$i$} & 1 & - & -1586.4 & -668.59 \\
\hline & 2 & -1995.7 & - & 737.04 \\
\hline & 3 & 916.15 & -548.36 & - \\
\hline
\end{tabular}

O.F. $=0.164, \sigma=7.40$.

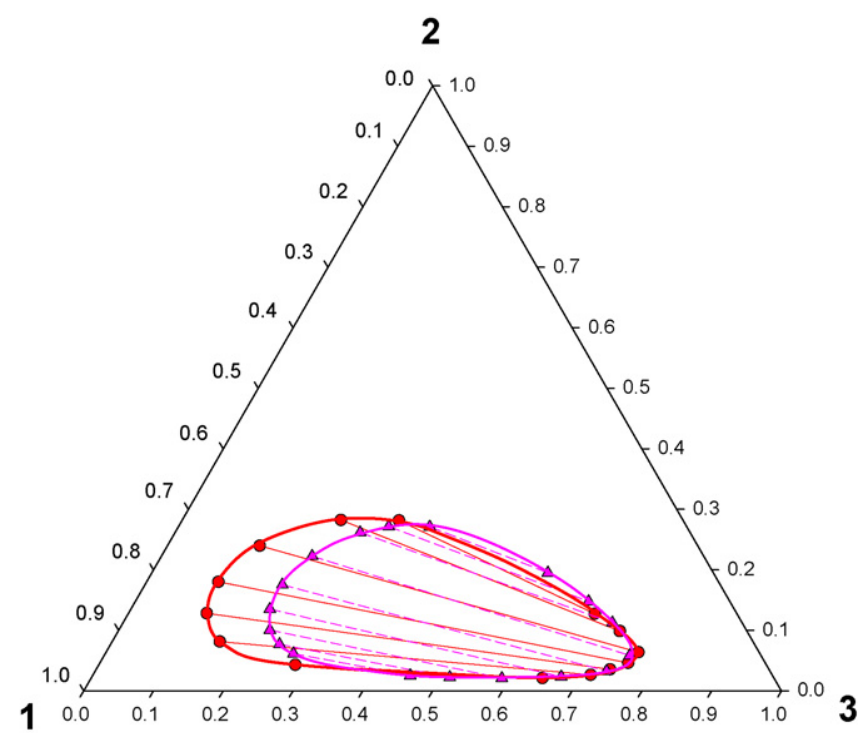

Fig. 9. Graphical comparison of the experimental ( $(\square-$ ) tie-lines using NRTL (molar fractions) for the water (1)+ dimethyl sulfoxide (2) + tetrahydrofuran (3) ternary system at $20^{\circ} \mathrm{C}$.

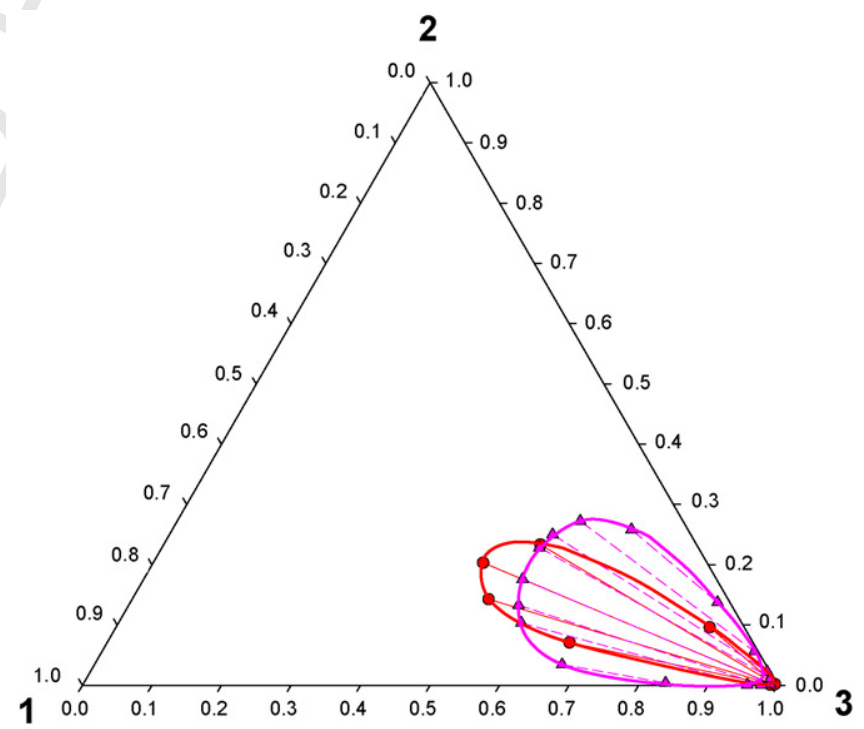

Fig. 10. Graphical comparison of the experimental ($(\triangle-)$ tie-lines using NRTL (molar fractions) for the trichloro acetic acid (1) + antipyrine (2) + water (3) ternary system at $30^{\circ} \mathrm{C}$.

of regressing LLE data for island and other complex types of systems.

\section{Conclusions}

A systematic study to evaluate the capability of the NRTL model to fit LLE data for island type ternary systems has been carried out. Analysis of the topological concepts related to the Gibbs energy of mixing surface, together with the Gibbs criterion of equilibrium based on the tangent plane, leads to the conclusion that the main requirement for the binary $g^{\mathrm{M}}$ curves 
calculated using NRTL model, to generate island type ternary systems, is that the $g^{\mathrm{M}}$ curve for one of the three binary systems must be much larger, or in other words, more negative (dissimilar binary $g^{\mathrm{M}}$ curve) than the other two binary $g^{\mathrm{M}}$ curves. In this sense, it is remarkable that the model cannot predict islands centred in the diagram, because this requires symmetry, and symmetry is not compatible with the requirement for NRTL to generate island type ternary systems. A relationship among the values of the binary parameters for the dissimilar and the other two binary pairs has also been determined, which has to be fulfilled in order to obtain an island type system. All this information has been used in an optimization program to correlate experimental LLE data for two island type ternary systems, proving that, although it is possible to obtain a certain correlation, the flexibility of the model is not enough to accurately correlate experimental data.

\section{Appendix A}

See Tables A1-A4.

Table A1

NRTL binary parameters $\left(A_{i j}, A_{j i}\right)$ for $x_{i, \min }=0.35$ and varying the $g_{\min }^{\mathrm{M}}$ value

\begin{tabular}{lcc}
\hline$g_{\min }^{\mathrm{M}}$ & $A_{i j}(\mathrm{~K})$ & $A_{j i}(\mathrm{~K})$ \\
\hline-0.2 & 399.43 & 271.16 \\
-0.25 & 517.82 & 116.34 \\
-0.3 & 689.17 & -42.895 \\
-2.98 & -2031.2 & 1306.4 \\
-3.0 & -2054.3 & 1430.6 \\
-3.1 & -2125.8 & 1776.9 \\
-3.3 & -2224.3 & 2281.2 \\
-3.4 & -2027.7 & 344.95 \\
-3.5 & -2049.2 & 261.58 \\
-4.0 & -2169.4 & -68.316 \\
-4.5 & -2297.4 & -328.31 \\
-5.0 & -2426.9 & -555.04 \\
\hline
\end{tabular}

Table A2

NRTL binary parameters $\left(A_{i j}, A_{j i}\right)$ for $x_{i, \min }=0.40$ and varying the $g_{\min }^{\mathrm{M}}$ value

\begin{tabular}{lcc}
\hline$g_{\min }^{\mathrm{M}}$ & $A_{i j}(\mathrm{~K})$ & $A_{j i}(\mathrm{~K})$ \\
\hline-0.2 & 358.62 & 304.64 \\
-0.25 & 405.80 & 194.07 \\
-0.3 & 480.46 & 73.651 \\
-0.4 & 733.42 & -182.93 \\
-0.5 & 1112.9 & -409.60 \\
-1.99 & -1436.5 & 846.19 \\
-2.0 & -1426.4 & 783.75 \\
-2.2 & -1416.6 & 385.09 \\
-2.4 & -1455.4 & 189.03 \\
-2.5 & -1479.4 & 110.44 \\
-3.0 & -1615.8 & -200.52 \\
-3.5 & -1760.4 & -446.96 \\
-4.0 & -1905.1 & -662.93 \\
-4.5 & -2047.8 & -860.69 \\
-5.0 & -2188.0 & -1046.2 \\
\hline
\end{tabular}

Table A3

NRTL binary parameters $\left(A_{i j}, A_{j i}\right)$ for $x_{i, \min }=0.45$ and varying the $g_{\min }^{\mathrm{M}}$ value

\begin{tabular}{lcc}
\hline$g_{\min }^{\mathrm{M}}$ & $A_{i j}(\mathrm{~K})$ & $A_{j i}(\mathrm{~K})$ \\
\hline-0.2 & 340.28 & 321.16 \\
-0.25 & 340.18 & 248.39 \\
-0.3 & 351.65 & 169.52 \\
-0.4 & 427.79 & -14.508 \\
-0.5 & 611.01 & -234.75 \\
-0.6 & 896.10 & -450.52 \\
-0.7 & 1234.9 & -623.45 \\
-0.8 & 1621.7 & -750.88 \\
-1.275 & -954.22 & 755.58 \\
-1.3 & -1027.2 & 948.95 \\
-1.4 & -1165.6 & 1317.1 \\
-1.5 & -1260.2 & 1609.8 \\
-1.6 & -1334.6 & 1897.9 \\
-1.7 & -1393.3 & 2208.6 \\
-1.8 & -1435.4 & 2575.5 \\
-1.9 & -1453.1 & 3083.6 \\
-2.0 & -997.83 & -204.01 \\
-2.1 & -1030.6 & -260.04 \\
-2.15 & -1047.2 & -286.98 \\
-2.2 & -1063.8 & -313.29 \\
-2.5 & -1164.5 & -460.77 \\
-3.0 & -1331.0 & -679.75 \\
-3.5 & -1492.9 & -877.94 \\
-4.0 & -1649.7 & -1062.5 \\
-4.5 & -1801.9 & -1237.4 \\
-5.0 & -1950.1 & -1404.9 \\
\hline & &
\end{tabular}

Table A4

NRTL binary parameters $\left(A_{i j}, A_{j i}\right)$ for $x_{i, \min }=0.50$ and varying the $g_{\min }^{\mathrm{M}}$ value

\begin{tabular}{lc}
\hline$g_{\min }^{\mathrm{M}}$ & $A_{i j}=A_{j i}(\mathrm{~K})$ \\
\hline-0.2 & 330.56 \\
-0.25 & 292.94 \\
-0.3 & 256.44 \\
-0.4 & 186.45 \\
-0.5 & 120.00 \\
-0.6 & 56.619 \\
-0.9 & -118.63 \\
-1.2 & -276.64 \\
-1.5 & -421.84 \\
-2.0 & -642.80 \\
-2.1 & -684.47 \\
-2.2 & -725.43 \\
-2.3 & -765.72 \\
-2.4 & -805.38 \\
-2.5 & -844.45 \\
-3.0 & -1032.0 \\
-3.5 & -1208.8 \\
-4.0 & -1377.3 \\
-4.5 & -1539.2 \\
-5.0 & -1695.8 \\
\hline
\end{tabular}

\section{References}

[1] R.E. Treybal, Liquid Extraction, second ed., McGraw-Hill, New York, 1963.

[2] A.W. Francis, Liquid-Liquid Equilibriums, John Wiley \& Sons, New York, 1963.

[3] F. Becker, P. Richter, Fluid Phase Equilib. 49 (1989) 157-166.

[4] D. Patterson, Polym. Eng. Sci. 22 (1982) 64-73.

[5] K. Řehák, J. Matouš, J.P. Novák, Fluid Phase Equilib. 109 (1995) 113-129. 
[6] J.P. Novák, K. Řehák, P. Voňka, J. Matouš, Fluid Phase Equilib. 208 (2003) 199-221.

[7] G.M. Wilson, J. Am. Chem. Soc. 86 (1964) 127-130.

[8] H. Renon, J.M. Prausnitz, AIChE J. 14 (1968) 135-144.

[9] J.M. Sørensen, W. Arlt, Liquid-Liquid Equilibrium Data Collection, Chemistry Data Series, Parts 2 and 4, vol. V, DECHEMA, Frankfurt, 1980.

[10] D. Abrams, J.M. Prausnitz, AIChE J. 21 (1975) 116-128.

[11] A. Marcilla, M.M. Olaya, J.A. Reyes-Labarta, A. Botella, Proceeedings of the 4th European Congress in Chemical Engineering (ECCE-4), Granada

(Spain), 2003.

[12] J.A. Reyes, J.A. Conesa, A. Marcilla, M.M. Olaya, Ind. Eng. Chem. Res. 40 (2001) 902-907.

[13] M.M. Olaya, A. Marcilla, M.D. Serrano, A. Botella, J.A. Reyes-Labarta, Ind. Eng. Chem. Res. 46 (2007) 7030-7037.

[14] J.V. Smith, R.W. Missen, W.R. Smith, AIChE J. 39 (1993) 707-710.

[15] Y. Jiang, W.R. Smith, G.R. Chapman, SIAM J. Control Optim. 5 (1995) 813-834.

Please cite this article in press as: M.M. Olaya, et al., Modelling liquid-liquid equilibria for island type ternary systems, Fluid Phase Equilib. (2008), doi:10.1016/j.fluid.2007.12.010 